\begin{tabular}{|c|c|}
\hline Title & Ultrahigh-speed multiplex coherent anti-Stokes Raman scattering microspectroscopy using scanning elliptical focal spot \\
\hline Author(s) & Kizawa, Shun; Hashimoto, Mamoru \\
\hline Citation & $\begin{array}{l}\text { The Journal of Chemical Physics, 155(14), } 144201 \\
\text { https://doi.org/10.1063/5.0063987 }\end{array}$ \\
\hline Issue Date & 2021-10-11 \\
\hline Doc URL & http:/hdl.handle.net/2115/82935 \\
\hline Rights & $\begin{array}{l}\text { This article may be downloaded for personal use only. A ny other use requires prior permission of the author and AIP } \\
\text { Publishing. This article appeared in J. Chem. Phys. 155, } 144201 \text { (2021) and may be found at } \\
\text { https://doi.org/10.1063/5.0063987 }\end{array}$ \\
\hline Type & article (author version) \\
\hline File Information & JCP21-A R-CHEM 2021-02524.pdf \\
\hline
\end{tabular}

Instructions for use 


\section{Ultra-high-speed multiplex coherent anti-Stokes Raman scattering microspectroscopy using scanning elliptical focal spot}

Shun Kizawa ${ }^{1, a)}$ and Mamoru Hashimoto ${ }^{2, b)}$

1) Division of Bioengineering and Bioinformatics, Graduate School of Information Science and Technology, Hokkaido University, North 14, West 9, Kita-ku, Sapporo 060-0814, Japan

2) Division of Bioengineering and Bioinformatics, Faculty of Information Science and Technology, Hokkaido University, North 14, West 9, Kita-ku, Sapporo 060-0814, Japan

(Dated: 14 September 2021)

We present a beam-scanning multiplex coherent anti-Stokes Raman scattering (CARS) microspectroscopy system using parallel excitation and parallel detection schemes based on an elliptical focal spot, which enables highly efficient signal acquisition even for short exposures. The elliptical focal spot was used to simultaneously observe the CARS signals of an enlarged region and reduce the peak irradiance. The developed system realized an acquisition rate of 34,139 spectra/s and enabled ultra-high-speed acquisition of a vibrational spectroscopic image covering the fingerprint region of $930-1830 \mathrm{~cm}^{-1}$ with $256_{(\mathrm{x})} \times 256_{(\mathrm{y})} \times 512_{\text {(spectrum) }}$ pixels in $1.92 \mathrm{~s}$ or with $128_{(\mathrm{x})} \times 128_{(\mathrm{y})} \times 256_{(\text {spectrum) }}$ pixels in $0.54 \mathrm{~s}$. We demonstrated ultra-high-speed hyperspectral imaging of a mixture of polymer beads in liquid linoleic acid and living adipocytes using the developed system. All of the present demonstrations were performed with low-peak-irradiance excitation of approximately $19 \mathrm{GW} / \mathrm{cm}^{2}$, which has been reported in previous studies to cause less photodamage to living cells. The label-free and ultra-high-speed identification and visualization of various molecules made possible by the present system will accelerate the development of practical live-cell investigation.

\footnotetext{
a) Present address: Hitachi Ltd, Research and Development Group, 1-280 Higashi-Koigakubo, Kokubunji, Tokyo, Japan

b) Author to whom correspondence should be addressed: hashimoto@ist.hokudai.ac.jp
} 


\section{INTRODUCTION}

Vibrational microspectroscopy based on Raman scattering allows us to identify various molecules and visualize their distribution without labels and has attracted attention in biological and medical applications ${ }^{1,2}$. Label-free analysis can be realized without prior investigation of the target molecules in the specimen because the molecular vibrational frequency is specific to the molecular species. In particular, molecular vibrations included in the fingerprint region $\left(600-1,800 \mathrm{~cm}^{-1}\right)$ contain a wealth of information about a lot of organic compounds and are useful for identifying intracellular biomolecules. However, living cells may change their morphological characteristics and intracellular biochemical reactions dynamically, and in addition, there is a concern that they will be damaged by laser irradiation ${ }^{3-11}$. Therefore, techniques that achieve high speed, high sensitivity, and low photoinduced damage are critical for practical live-cell analysis.

Barely satisfying these requirements, spontaneous Raman scattering microspectroscopy with parallel excitation and parallel detection schemes has been developed, including a multifocus confocal method ${ }^{12}$ and a line-scan method ${ }^{13,14}$. The parallel excitation and detection scheme increases the exposure time per point, allowing a reduction in the total acquisition time compared with a single-point excitation and detection scheme with the same irradiance. However, the spectral acquisition rates of these methods are restricted to $40 \mathrm{spectra} / \mathrm{s}^{12}$ and 267 spectra/s ${ }^{14}$, respectively, because spontaneous Raman scattering is typically extremely weak. To accelerate biological studies such as monitoring of fast intracellular dynamics and high-throughput screening, there is a strong demand for a faster vibrational microspectroscopic modality.

Coherent Raman scattering (CRS), such as coherent anti-Stokes Raman scattering (CARS) and stimulated Raman scattering (SRS), in which specific molecular vibrations are coherently excited, can offer a Raman signal level orders of magnitude higher than spontaneous Raman scattering and is advantageous for high-speed observation ${ }^{15-17}$. In particular, single-frequency CRS imaging in which a specific band is selectively excited allows extremely high-speed imaging beyond video-rates ${ }^{18-20}$. With the aim of minimizing photoinduced damage and achieving faster acquisition in CRS microscopy, the previous study demonstrated that parallel excitation and parallel detection schemes using multifocus scanning allowed highly efficient measurement with lower photoinduced damage than 
single-focus scanning ${ }^{10,21}$. This is because photoinduced damage of living cells in CRS microscopy is primarily caused by the high peak irradiance of the excitation laser light rather than its energy. However, despite the high-speed capability, single-band CRS microscopy has the drawback that it does not provide sufficient information to distinguish molecular compositions with overlapping spectra within complicated heterogeneous specimens.

Broadband CRS microspectroscopy, in which every molecular vibration within the fingerprint region is excited, offers vibrational spectroscopic images similar to spontaneous Raman scattering microspectroscopy and is therefore useful for more detailed investigations of biological specimens. Among several kinds of broadband CRS microscopic techniques ${ }^{22-27}$, high-speed capability covering most of the fingerprint region has been demonstrated, such as 1,250 spectra/s with multiplex CARS ${ }^{23}, 10,000$ spectra/s with multiplex $\mathrm{SRS}^{25}$, and 24,000 spectra/s with FT-CARS ${ }^{27}$. However, these techniques involved high-peak-irradiance excitation $\left(240 \mathrm{GW} / \mathrm{cm}^{2}\right.$ with multiplex $\mathrm{CARS}^{23}, 520 \mathrm{GW} / \mathrm{cm}^{2}$ with multiplex $\mathrm{SRS}^{25}$, and 12.8 $\mathrm{TW} / \mathrm{cm}^{2}$ with FT-CARS ${ }^{27}$ ), and may cause photoinduced severe cell damage associated with multiphoton events ${ }^{3-11}$.

Here, we present a multiplex CARS microspectroscopy system using parallel excitation and parallel detection schemes. The concept of the system is a combination of beam-scanning multiplex CARS microspectroscopy ${ }^{28}$, a parallel excitation scheme using an elliptical focal spot, and a parallel detection scheme using an electron-multiplying charge-coupled device camera (EM-CCD). Our system realized ultra-high-speed hyperspectral CARS imaging with an acquisition rate of 34,139 spectra/s and a low peak irradiance excitation of $19 \mathrm{GW} / \mathrm{cm}^{2}$.

\section{DESIGN OF SYSTEM}

Figure 1 (a) presents a schematic diagram of the developed CARS microspectroscopy system, which consisted of two synchronized lasers ${ }^{19,29}$, an elliptical beam shaper, a onedimensional beam-scanner, a microscope, and a spectrometer with a two-dimensional image sensor (see Sec. I of the supplementary material). The developed microspectroscopy system used a combination of multiplex CARS spectroscopy and beam-scanning of an elliptical focal spot for parallel excitation and detection. In multiplex CARS spectroscopy, many molecular vibrations are simultaneously excited by the energy difference between a narrowband laser beam $\left(\omega_{1}\right.$ beam) and a broadband laser beam $\left(\omega_{2}\right.$ beam $)$, and then an anti-Stokes 
Raman scattering spectrum induced by the narrowband laser beam is observed with a spectrometer $^{30}$. Beam-scanning multiplex CARS microspectroscopy is an imaging technique for observing CARS spectra on a line by scanning a beam spot along the slit of a spectrometer and by detecting spatially resolved CARS spectra using a two-dimensional image sensor ${ }^{28}$. An elliptical focal spot, whose major axis is along the beam-scanning direction, is utilized to simultaneously observe the CARS signals of the enlarged region and reduce the peak irradiance.

We employed synchronized picosecond (ps Ti:S, $\omega_{1}$ ) and femtosecond Ti:sapphire (fs Ti:S, $\left.\omega_{2}\right)$ lasers as light sources ${ }^{31-33}$. The signal intensity of CARS generated by a third-order nonlinear optical effect is proportional to the product of the square of the peak irradiance of the $\omega_{1}$ beam and the peak irradiance of $\omega_{2}$ beam. Therefore, high-power lasers are required to compensate for the reduction in peak irradiance due to the enlarged elliptical focal spot. In the present system, the maximum powers of the $\omega_{1}$ and $\omega_{2}$ beams were $200 \mathrm{~mW}$ and 100 $\mathrm{mW}$ on the sample plane, respectively. The spectral resolution and observable spectral range are determined by the spectral bandwidth of the narrowband $\omega_{1}$ beam and the broadband $\omega_{2}$ beam, respectively. Figure 1 (b) shows the spectra of the laser sources. The bandwidth of the $\omega_{1}$ beam was $0.11 \mathrm{~nm}$ at $730 \mathrm{~nm}$, and the bandwidth of the $\omega_{2}$ beam was $80 \mathrm{~nm}$ centered at $800 \mathrm{~nm}$. As a result, the system covered the fingerprint region $\left(580-1,830 \mathrm{~cm}^{-1}\right)$ with a spectral resolution of $2.0 \mathrm{~cm}^{-1}$. Although the femtosecond laser pulses had high peak power at the output because of their short duration of $12 \mathrm{fs}$, the dispersion of optical components stretched the duration and reduced the peak power. Figure 1 (c) shows the intensity auto-correlation signals of the two laser beams on the focal plane (see Sec. II of the supplementary material). The full width at half maximums (FWHMs) of the intensity auto-correlation signals were 7.68 ps for the $\omega_{1}$ beam and 2.37 ps for the $\omega_{2}$ beam, which corresponded to pulse durations of $4.99 \mathrm{ps}$ and $1.54 \mathrm{ps}$, respectively.

The developed system utilized an elliptical focal spot with an ellipticity of 0.125 for parallel excitation and detection. In slit confocal Raman scattering microspectroscopy, a line-shaped focal spot is used for parallel excitation ${ }^{13,14}$. However, such significant deformation of the focal spot greatly lowers the peak irradiance, considerably reducing the signals of CARS, which is a nonlinear optical phenomenon. To investigate living cells at high speed, it is essential to use light sources with the highest peak irradiance within a safe operating range. For instance, König et al. reported that a peak irradiance higher than $20 \mathrm{GW} / \mathrm{cm}^{2}$ 
affects the cloning efficiency of cells ${ }^{3-6}$. The total peak irradiance can be estimated by:

$$
P=\left\{\frac{I_{1}}{\tau_{1} f_{\text {rep }} \pi\left(\frac{0.61 \lambda_{1}}{N A}\right)^{2}}+\frac{I_{2}}{\tau_{2} f_{\text {rep }} \pi\left(\frac{0.61 \lambda_{2}}{N A}\right)^{2}}\right\} \epsilon
$$

where $I_{1}$ and $I_{2}$ are the averaged laser powers of the $\omega_{1}$ and $\omega_{2}$ beams, $\tau_{1}$ and $\tau_{2}$ are the pulse durations of the $\omega_{1}$ and $\omega_{2}$ beams, $f_{\text {rep }}$ is the repetition frequency of the pulses, $\lambda_{1}$ and $\lambda_{2}$ are the wavelengths of the $\omega_{1}$ and $\omega_{2}$ beams, and $\epsilon$ is the ellipticity ${ }^{9}$. From the experimental conditions (see Sec. I of the supplementary material), we determined the ellipticity to be 0.125, to satisfy the safety threshold of photo-induced damage with a peak irradiance of approximately $19 \mathrm{GW} / \mathrm{cm}^{2}$. An elliptical focal spot with an ellipticity of 0.125 was formed by focusing an elliptical beam with an ellipticity of 0.125, shown in Fig. 1 (d). A pair of cylindrical lenses converted the Gaussian beam from the laser to an elliptical beam.

For higher speed imaging, it is desirable to simultaneously obtain the spectra on a long line by using a two-dimensional image sensor, similar to slit confocal Raman scattering microspectrocopy ${ }^{13,14}$ and beam-scanning multiplex CARS microspectroscopy ${ }^{28}$. Hence, we combined beam-scanning multiplex CARS microspectroscopy and an elliptical focal spot. Furthermore, an EM-CCD was used for the two-dimensional image sensor to perform highspeed readout. Due to these schemes, spectral information with a bandwidth of $900 \mathrm{~cm}^{-1}$ on a beam-scanning line of $68.3 \mu \mathrm{m}$ could be acquired in $7.5 \mathrm{~ms}$ (frame transfer time, 0.3 ms; exposure time, $7.2 \mathrm{~ms}$; data size, $256_{(y)} \times 512_{\text {(spectrum) }}$ pixels) or $4.2 \mathrm{~ms}$ (frame transfer time, $0.3 \mathrm{~ms}$; exposure time, $3.9 \mathrm{~ms}$; data size, $128_{(y)} \times 256_{(\text {spectrum })}$ pixels) with $2 \times 2$ camera binning. Here, it should be noted that the minimum exposure time is determined by the minimum readout time, and signal reading is not possible during the frame transfer operation. To realize nearly uniform illumination on the scanning line, an integer multiple of a half cycle of the beam scanning must be adjusted to the exposure time of the camera. For example, the beam scanning period was adjusted to $2.06 \mathrm{~ms}$ for an exposure time of $7.2 \mathrm{~ms}$ (3.5 scans/exposure) and to $2.60 \mathrm{~ms}$ for an exposure time of $3.9 \mathrm{~ms}$ (1.5 scans/exposure). The insertion image in the upper left of Fig. 1 (a) is an example of a single shot taken by the EM-CCD, i.e., the CARS spectral distribution along the beam scanning line. A sample of mixed beads consisting of polystyrene (PS) and polymethylmethacrylate (PMMA) was observed. The strong signal in the top horizontal bar corresponds to a PS bead, and the weak signals in the other horizontal bars correspond to PMMA beads. As shown in Fig. 1 (d), the elliptical focal spot was shaped to be about $0.5 \mu \mathrm{m}$ in the minor axis and about 
$4.0 \mu \mathrm{m}$ in the major axis. In contrast, the beam scan line was about $78.1 \mu \mathrm{m}$, and the observation range was $68.3 \mu \mathrm{m}$ (see Sec. I of the supplementary material). The intensity distribution along the long axis of the elliptical focus is averaged by beam scanning, and uniform line signals are obtained by out-of-ranging at both ends where the beam stops.

\section{RESULTS}

\section{A. Hyperspectral CARS imaging of polymer beads}

We demonstrate ultra-high-speed hyperspectral CARS imaging with a spectral acquisition rate of 34,139 spectra/s to decompose the spatial distribution of substances with micrometerorder dimensions. Figure 2 shows the observed result for a mixture of PS and polyethylene (PE) beads in liquid linoleic acid (LA) (see Sec. III. 1 of the supplementary material). The observed $256 \times 256$ CARS spectra were individually converted to $\operatorname{Im}\left[\chi^{(3)}\right]$ spectra using maximum entropy method (MEM) ${ }^{34-36}$ (see Sec. IV and Movie S1 of the supplementary material). Figure 2 (a-c) shows the $\operatorname{Im}\left[\chi^{(3)}\right]$ images at $1,001 \mathrm{~cm}^{-1}, 1,129 \mathrm{~cm}^{-1}$, and 1,654 $\mathrm{cm}^{-1}$. Figure $2(\mathrm{~d})$ shows the $\operatorname{Im}\left[\chi^{(3)}\right]$ spectra at the red, green and blue arrowheads in Fig. 2 (a-c). These three spectra contained different bands. From the shape of the image, the bands of $1,001,1,129$, and $1,654 \mathrm{~cm}^{-1}$ were assigned to the phenyl ring breathing mode of PS, the skeletal tans $\mathrm{C}-\mathrm{C}$ stretching mode of $\mathrm{PE}$, and the $\mathrm{C}=\mathrm{C}$ stretching mode of $\mathrm{LA}$, respectively. Figure 2 (e) shows an $\operatorname{Im}\left[\chi^{(3)}\right]$ spectral profile on a line along the beam-scanning direction, which was obtained from a single frame of the EM-CCD image sensor. In spite of the ultrahigh spectral acquisition rate of 34,139 spectra/s, the detailed structure of every spectrum could be identified with sufficient sensitivity. Incidentally, dark spots on the largest bead at the center are due to the beads in out-of-focus positions.

We performed multivariate curve resolution alternating least squares (MCR-ALS) anal$y_{s i s^{37-39}}$ of the obtained hyperspectral $\operatorname{Im}\left[\chi^{(3)}\right]$ image to decompose the constituent components using both spatial and spectral information (see Sec. IV of the supplementary material). Figure 3 shows decomposed spectra (a-c) and their spatial distributions (d-f). The spectral components (SC) 1-3 corresponded to PS, PE, and LA, respectively, and could be separated into pure components. From MCR analysis, PS and PE were completely separated and the concentration distribution of LA was also obtained. The concentration of 
LA in PS was higher than that in PE. This difference in LA concentration in each bead was caused by LA's higher solubility to PS than that of $\mathrm{PE}^{40,41}$.

\section{B. Hyperspectral CARS imaging of living cells}

We demonstrated the ultra-high-speed hyperspectral CARS imaging of living cells with an acquisition rate of 34,139 spectra/s, as shown in Fig. 4. The observed cells were 3T3L1 adipocytes incubated with oleic acid or linoleic acid for 10 days (see Sec. III. 2 of the supplementary material). The adipocytes metabolize introduced fatty acids and store them in lipid droplets (LDs) as a triglyceride. Oleic acid and linoleic acid both have the same carbon number; however, the former contains one double bond, and the latter contains two double bonds. Figure 4 (a) and (c) show $\operatorname{Im}\left[\chi^{(3)}\right]$ images of a sample mixed with oleic acid and a sample mixed with linoleic acid, respectively, reconstructed with the $\mathrm{CH}_{2}$ scissoring mode around $1,438 \mathrm{~cm}^{-1}$. Figure $4(\mathrm{~b})$ and $(\mathrm{d})$ show $\operatorname{Im}\left[\chi^{(3)}\right]$ images of a sample incubated in a medium containing oleic acid and a sample incubated in a medium containing linoleic acid, respectively, reconstructed with the $\mathrm{C}=\mathrm{C}$ stretching mode around $1,654 \mathrm{~cm}^{-1}$. In both images, lipid droplets were clearly visualized. Figure 4 (e) shows $\operatorname{Im}\left[\chi^{(3)}\right]$ spectra at a single pixel in the LDs formed on each sample. Since the degree of unsaturation can be evaluated with the ratio of the $\mathrm{CH}_{2}$ scissoring mode around $1,438 \mathrm{~cm}^{-1}$ and the $\mathrm{C}=\mathrm{C}$ stretching mode around $1,654 \mathrm{~cm}^{-1}{ }^{42}$ we applied this approach to the obtained data, as shown in Fig. 4 $(f, g)$. These results indicate that the adipocytes incubated with linoleic acid accumulates LDs with a higher degree of unsaturation than that of the adipocytes incubated with oleic acid. The difference in the chemical composition of the lipid droplets due to the feeding of different fatty acids is also reflected in the $=\mathrm{C}-\mathrm{H}$ bending mode around $1,260 \mathrm{~cm}^{-1}$ as shown in Fig. $4(\mathrm{e})^{43}$. In addition, no morphological changes due to the photoinduced damage were observed.

We also obtained a hyperspectral CARS image of living adipocytes incubated with oleic acid in $0.54 \mathrm{~s}$ by utilizing a $2 \times 2$ binning setting, as shown in Fig. 5 . Figure 5 (a) shows the reconstructed $\operatorname{Im}\left[\chi^{(3)}\right]$ image at $1,438 \mathrm{~cm}^{-1}$, in which the LDs were visualized with high contrast. Figure 5 (b) shows an $\operatorname{Im}\left[\chi^{(3)}\right]$ spectrum at the arrowheads in Fig. 5 (a). Although the number of spectral pixels was 256, the spectral structure specific to the LDs could be identified. Figure 5 (c) shows an $\operatorname{Im}\left[\chi^{(3)}\right]$ spectral profile on a line along the beam-scanning 
direction. Despite the fact that the beam was scanned for only 1.5 reciprocations during one exposure of $3.9 \mathrm{~ms}$, the spatial distributions of every spectrum on the line along the beam-scanning direction were obtained with sufficient sensitivity.

The shoulder band of about $1480 \mathrm{~cm}^{-1}$ shown in Fig. 5 (b) seems to be related to the lipid membrane. This band does not appear in Fig. 4 (e). We attribute this to the difference in focusing position in the optical axis direction.

\section{DISCUSSION}

The developed ultra-high-speed multiplex CARS microspectroscopy system with lowpeak-irradiance excitation has high potential to accelerate the development of practical live-cell investigation, such as regenerative medicine ${ }^{44}$, intraoperative rapid diagnosis ${ }^{45,46}$, and imaging flow cytometry ${ }^{47}$. The system operated with a spectral acquisition rate of 34,139 spectra/s under low-peak-irradiance excitation of $19 \mathrm{GW} / \mathrm{cm}^{2}$. Compared with the previously reported fastest vibrational spectroscopy system ${ }^{48}$, this spectral acquisition rate is $1 / 3$ times. The spectral acquisition rate of the present system was limited due to the pixel readout speed of $22 \mathrm{MHz}$. However, by utilizing an EM-CCD with a faster reading speed of 30 $\mathrm{MHz}^{49}$, a spectral acquisition rate of up to 64,256 spectra/s with $256_{(\mathrm{x})} \times 256_{(\mathrm{y})} \times 256_{(\text {spectrum })}$ pixels should be possible easily. In addition, by using an EM-CCD with 8 output split frame transfer at $13.6 \mathrm{MHz}$ reading speed, a spectral acquisition rate of up to 496,080 spectra/s with $240_{(\mathrm{x})} \times 240_{(\mathrm{y})} \times 240_{(\text {spectrum) }}$ pixels would also be realized ${ }^{50}$. On the other hand, even if the spectral acquisition with the system is sufficiently fast, the spectral processing using MEM is time-consuming. The computing time required for MEM analysis per spectrum was about $10 \mathrm{~ms}$ in our environment. It took about 10 minutes to quantitatively analyze a spectral image of $256_{(\mathrm{x})} \times 256_{(\mathrm{y})} \times 256_{\text {(spectrum) }}$ pixels. The use of deep learning is one of the methods to solve this problem. A well-trained deep learning model can retrieve $\operatorname{Im}\left[\chi^{(3)}\right]$ from individual CARS spectra in $0.1 \mathrm{~ms}^{51}$, which has the potential for real-time spectral imaging.

As for the peak irradiance, the relationship between photoinduced damage and peak irradiance in nonlinear optical microscopy with near-infrared ultrashort-pulse lasers at wavelengths around 700-900 $\mathrm{nm}$ has been investigated in a wide range of peak irradiances: below $20 \mathrm{GW} / \mathrm{cm}^{2}$ (safety operation ${ }^{3-6}$ ), at $20-100 \mathrm{GW} / \mathrm{cm}^{2}$ (decrease in cloning efficiency ${ }^{3-6}$, im- 
paired regulation of cell membrane molecular transport ${ }^{10}$ ), at $100-200 \mathrm{GW} / \mathrm{cm}^{2}$ (plasma membrane blebbing and myelin sheath damage ${ }^{8}$, morphological cell damage ${ }^{6}$ ), and up to 1 $\mathrm{TW} / \mathrm{cm}^{2}$ (complete cell fragmentation ${ }^{11}$ ). From these thresholds, we believe that the peak irradiance excitation of $19 \mathrm{GW} / \mathrm{cm}^{2}$ in the present system is suitable for safe live-cell observation. Higher peak irradiance may also be available using deep-near-infrared excitation with wavelengths longer than $1000 \mathrm{~nm}$, considered less cell-damaging.

Our system can detect sufficient CARS signals even with low peak irradiance excitation and short exposure time. This advantage was made possible by using the EM-CCD and parallel measurement using the elliptical focal spot. The EM-CCD has a mechanism to amplify weak signals, which allows us to observe weak CARS signals at high speed. The elliptical beam-scanning system is also applicable to multiplex SRS. In this case, the EMCCD cannot be used because SRS requires lock-in detection. The detector for the SRS needs high dynamic range rather than high sensitivity. The SRS signal is enhanced by heterodyne mixing with the excitation beam, which causes a strong background. A two-dimensional lock-in camera, which equips high dynamic range capability even under intense background, would be helpful for multiplex SRS detection ${ }^{52,53}$.

Our system also offers rich spectral information covering the fingerprint region with a sufficient number of spectral pixels. Therefore, MCR-ALS analysis of the obtained hyperspectral image is quite useful for the investigation of spectrally overlapping molecular compositions within complicated heterogeneous specimens. Although the observable spectral bandwidth of the present system was limited to $900 \mathrm{~cm}^{-1}$ in the range of $580-1,830$ $\mathrm{cm}^{-1}$, by selecting an appropriate diffraction grating for the spectrometer, entire fingerprint region of $580-1,830 \mathrm{~cm}^{-1}$ can be covered. In terms of the bandwidth performance, an ultrabroadband laser source will make it possible to obtain ultra-broadband hyperspectral CARS images covering over $3,000 \mathrm{~cm}^{-1}$, in which high-wave number region and silent region are also included ${ }^{23,24,54}$. In addition, spectral distortion caused by the non-resonant background can be reduced by adopting the epi-detection scheme ${ }^{55}$ and polarization techniques ${ }^{56}$. The epi-detection scheme can also expand applicational capability, such as pathological analysis of large area thick tissue, endoscopy, and in vivo imaging. We expect that non-resonant background-free techniques combined with our concept, in which parallel excitation and detection allow high speed and minimal photoinduced damage, will provide many more interesting biochemical components besides lipid droplets. 


\section{CONCLUSION}

In conclusion, we developed a multiplex CARS microspectroscopy system using a scanning elliptical focal spot. The elliptical focal spot, whose major axis was along the slit of a spectrometer, was utilized for simultaneously observing the CARS signals of an enlarged region and for reducing the peak irradiance. By scanning the elliptical focal spot along the slit of the spectrometer and simultaneously obtaining the spectra on the beamscanning trajectory using the EM-CCD, ultra-high-speed hyperspectral CARS imaging with low-peak-irradiance excitation was achieved. We obtained a hyperspectral CARS image $\left(256_{(\mathrm{x})} \times 256_{(\mathrm{y})} \times 512_{\text {(spectrum) }}\right.$ pixels) covering $900 \mathrm{~cm}^{-1}$ within fingerprint region in $1.92 \mathrm{~s}$ with a spectral acquisition rate of 34,139 spectra/s using a mixture of PS and PE beads in liquid LA and living 3T3-L1 adipocytes as specimens. We also succeed in acquiring a hyperspectral CARS image $\left(128_{(\mathrm{x})} \times 256_{(\mathrm{y})} \times 256_{\text {(spectrum) }}\right.$ pixels $)$ of living 3T3-L1 adipocytes $900 \mathrm{~cm}^{-1}$ within fingerprint region in $0.54 \mathrm{~s}$ with a spectral acquisition rate of 30,485 spectra/s. All observations were always performed with low-peak-irradiance excitation of 19 $\mathrm{GW} / \mathrm{cm}^{2}$, which is the highest peak irradiance within the safe operating range of nonlinear optical microscopy. The presented vibrational microspectroscopy would be beneficial in the investigation of living cells.

\section{AUTHOR CONTRIBUTIONS}

S. K. developed the imaging system, performed all experiments, and analyzed all data. S. K. and M. H. conceived the idea and wrote the manuscript. M. H. developed the light source and supervised the project.

\section{SUPPLEMENTARY MATERIAL}

See the supplementary material for the complete experimental system, detail of the intensity auto-correlator, sample preparation, data processing, and the movie legend. 


\section{ACKNOWLEDGMENTS}

The authors thank Hamamatsu Photonics K.K. for supporting of the EM-CCD. The authors also thank Kyotaro Horio (Department of Bioengineering, School of Engineering, The University of Tokyo), Naoki Yamato and Ryuji Abe (Graduate School of Information Science and Technology, Hokkaido University) for their supports in the sample preparation. This work is supported by JSPS KAKENHI Grant Number JP21H04950.

\section{DATA AVAILABILITY}

The data that support the findings of this study are available from the corresponding author upon reasonable request.

\section{REFERENCES}

${ }^{1}$ J.-X. Cheng and X. S. Xie, Science 350, aaa8870 (2015).

${ }^{2}$ B. Kann, H. L. Offerhaus, M. Windbergs, and C. Otto, Adv. Drug Deliv. Rev. 89, 71 (2015).

${ }^{3}$ K. König, H. Liang, M. Berns, and B. Tromberg, Nature 377, 20 (1995).

${ }^{4}$ K. König, H. Liang, M. Berns, and B. Tromberg, Opt. Lett. 21, 1090 (1996).

${ }^{5}$ K. König, P. So, W. Mantulin, and E. Gratton, Opt. Lett. 22, 135 (1997).

${ }^{6}$ K. König, T. Becker, P. Fischer, I. Riemann, and K.-J. Halbhuber, Opt. Lett. 24, 113 (1999).

${ }^{7}$ A. Hopt and E. Neher, Biophys. J. 80, 2029 (2001).

${ }^{8}$ Y. Fu, H. Wang, R. Shi, and J.-X. Cheng, Opt. Express 14, 3942 (2006).

${ }^{9}$ R. Galli, O. Uckermann, E. F. Andresen, K. D. Geiger, E. Koch, G. Schackert, G. Steiner, and M. Kirsch, PloS one 9, e110295 (2014).

${ }^{10}$ T. Minamikawa, Y. Murakami, N. Matsumura, H. Niioka, S. Fukushima, T. Araki, and M. Hashimoto, J. Spectrosc. 2017 (2017).

${ }^{11}$ A. Vogel, J. Noack, G. Hüttman, and G. Paltauf, Appl. Phys. B 81, 1015 (2005).

${ }^{12}$ M. Okuno and H. Hamaguchi, Opt. Lett. 35, 4096 (2010).

${ }^{13}$ K. A. Christensen and M. D. Morris, Appl. Spectrosc. 52, 1145 (1998).

${ }^{14}$ J. Qi and W.-C. Shih, Appl. Opt. 53, 2881 (2014). 
${ }^{15}$ A. Zumbusch, G. R. Holtom, and X. S. Xie, Phys. Rev. Lett. 82, 4142 (1999).

${ }^{16}$ M. Hashimoto, T. Araki, and S. Kawata, Opt. Lett. 25, 1768 (2000).

${ }^{17}$ H. Rigneault and P. Berto, APL Photonics 3, 091101 (2018).

${ }^{18}$ B. G. Saar, C. W. Freudiger, J. Reichman, C. M. Stanley, G. R. Holtom, and X. S. Xie, Science 330, 1368 (2010).

${ }^{19}$ H. Cahyadi, J. Iwatsuka, T. Minamikawa, H. Niioka, T. Araki, and M. Hashimoto, J. Biomed. Opt. 18, 096009 (2013).

${ }^{20}$ Y. Wakisaka, Y. Suzuki, O. Iwata, A. Nakashima, T. Ito, M. Hirose, R. Domon, M. Sugawara, N. Tsumura, H. Watarai, et al., Nat. Microbiol. 1, 16124 (2016).

${ }^{21}$ T. Minamikawa, M. Hashimoto, K. Fujita, S. Kawata, and T. Araki, Opt. Express 17, 9526 (2009).

${ }^{22}$ D. Polli, V. Kumar, C. M. Valensise, M. Marangoni, and G. Cerullo, Laser Photonics Rev. 12, 1800020 (2018).

${ }^{23}$ H. Kano, T. Maruyama, J. Kano, Y. Oka, D. Kaneta, T. Guerenne, P. Leproux, V. Couderc, and M. Noguchi, OSA Continuum 2, 1693 (2019).

${ }^{24}$ C. H. Camp Jr, Y. J. Lee, J. M. Heddleston, C. M. Hartshorn, A. R. H. Walker, J. N. Rich, J. D. Lathia, and M. T. Cicerone, Nat. Photon. 8, 627 (2014).

${ }^{25}$ L. Czerwinski, J. Nixdorf, G. Di Florio, and P. Gilch, Opt. Lett. 41, 3021 (2016).

${ }^{26}$ A. S. Duarte, C. Schnedermann, and P. Kukura, Sci. Rep. 6, 37516 (2016).

${ }^{27}$ R. Kinegawa, K. Hiramatsu, K. Hashimoto, V. R. Badarla, T. Ideguchi, and K. Goda, J. Raman Spectrosc. 50, 8 (2019).

${ }^{28}$ I. S. Ryu, C. H. Camp, Y. Jin, M. T. Cicerone, and Y. J. Lee, Opt. Lett. 40, 5826 (2015).

${ }^{29}$ T. Minamikawa, N. Tanimoto, M. Hashimoto, T. Araki, M. Kobayashi, K. Fujita, and S. Kawata, Appl. Phys. Lett. 89, 191101 (2006).

${ }^{30}$ S. Kröll, M. Aldén, T. Berglind, and R. J. Hall, Appl. Opt. 26, 1068 (1987).

${ }^{31}$ M. Müller and J. M. Schins, J. Phys. Chem. B 106, 3715 (2002).

${ }^{32}$ J.-x. Cheng, A. Volkmer, L. D. Book, and X. S. Xie, J. Phys. Chem. B 106, 8493 (2002).

${ }^{33}$ R. Selm, M. Winterhalder, A. Zumbusch, G. Krauss, T. Hanke, A. Sell, and A. Leitenstorfer, Opt. Lett. 35, 3282 (2010).

${ }^{34}$ E. M. Vartiainen, J. Opt. Soc. Am. B 9, 1209 (1992).

${ }^{35}$ E. M. Vartiainen, H. A. Rinia, M. Müller, and M. Bonn, Opt. Express 14, 3622 (2006).

${ }^{36}$ H. A. Rinia, M. Bonn, M. Müller, and E. M. Vartiainen, ChemPhysChem 8, 279 (2007). 
${ }^{37}$ L. Zhang, M. J. Henson, and S. S. Sekulic, Anal. Chim. Acta 545, 262 (2005).

${ }^{38}$ M. Ando and H. Hamaguchi, J. Biomed. Opt. 19, 011016 (2013).

${ }^{39}$ D. Zhang, P. Wang, M. N. Slipchenko, D. Ben-Amotz, A. M. Weiner, and J.-X. Cheng, Analytical chemistry 85, 98 (2013).

${ }^{40}$ C. M. Hansen, Hansen solubility parameters: a user's handbook (CRC press, 2007).

${ }^{41}$ A. De La Peña-Gil, J. F. Toro-Vazquez, and M. A. Rogers, Food Biophysics 11, 283 (2016).

${ }^{42}$ H. A. Rinia, K. N. Burger, M. Bonn, and M. Müller, Biophys. J. 95, 4908 (2008).

${ }^{43}$ I. W. Schie, L. Nolte, T. L. Pedersen, Z. Smith, J. Wu, I. Yahiatene, J. W. Newman, and T. Huser, Analyst 138, 6662 (2013).

${ }^{44}$ A. Ghita, F. C. Pascut, V. Sottile, C. Denning, and I. Notingher, EPJ techniques and instrumentation 2, 6 (2015).

${ }^{45}$ T. C. Hollon, S. Lewis, B. Pandian, Y. S. Niknafs, M. R. Garrard, H. Garton, C. O. Maher, K. McFadden, M. Snuderl, A. P. Lieberman, et al., Cancer Res. 78, 278 (2018).

${ }^{46}$ K. S. Shin, A. T. Francis, A. H. Hill, M. Laohajaratsang, P. J. Cimino, C. S. Latimer, L. F. Gonzalez-Cuyar, L. N. Sekhar, G. Juric-Sekhar, and D. Fu, Sci. Rep. 9, 1 (2019).

${ }^{47}$ Y. Suzuki, K. Kobayashi, Y. Wakisaka, D. Deng, S. Tanaka, C.-J. Huang, C. Lei, C.-W. Sun, H. Liu, Y. Fujiwaki, et al., PNAS, Proc. Natl. Acad. Sci. USA 116, 15842 (2019).

${ }^{48}$ R. Kameyama, S. Takizawa, K. Hiramatsu, and K. Goda, ACS Photonics 8, 975 (2020). 49 "Andor iXon Ultra 888 specifications," https://andor.oxinst.com/assets/uploads/ products/andor/documents/andor-ixon-ultra-emccd-specifications.pdf, accessed: 2021-07-14.

50 "First Light Imaging OCAM ${ }^{2}$ k," https://www.tokyoinst.co.jp/product_file/file/ FL01_cat02_ja.pdf, accessed: 2021-07-14.

${ }^{51}$ C. Valensise, A. Giuseppi, F. Vernuccio, A. De la Cadena, G. Cerullo, and D. Polli, APL Photonics 5, 061305 (2020).

${ }^{52}$ D. Lioe, K. Mars, S. Kawahito, K. Yasutomi, K. Kagawa, T. Yamada, and M. Hashimoto, Sensors 16, 532 (2016).

${ }^{53}$ K. Mars, D. X. Lioe, S. Kawahito, K. Yasutomi, K. Kagawa, T. Yamada, M. Hashimoto, et al., Sensors 17, 2581 (2017).

${ }^{54}$ H. Yoneyama, K. Sudo, P. Leproux, V. Couderc, A. Inoko, and H. Kano, APL Photonics 3, 092408 (2018). 
${ }^{55}$ J.-X. Cheng, A. Volkmer, L. D. Book, and X. S. Xie, J. Phys. Chem. B 105, 1277 (2001). ${ }^{56}$ J.-X. Cheng, L. D. Book, and X. S. Xie, Opt. Lett. 26, 1341 (2001). 

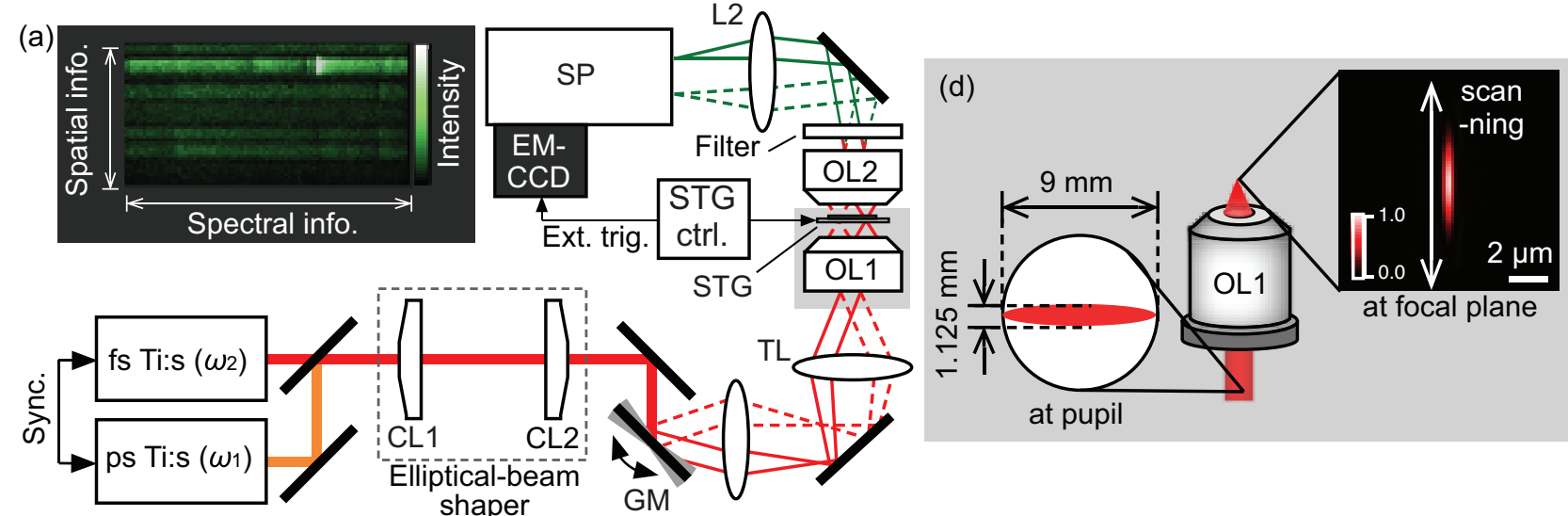

- $\omega_{1}$ laser $-\omega_{2}$ laser - CARS signal

(b)

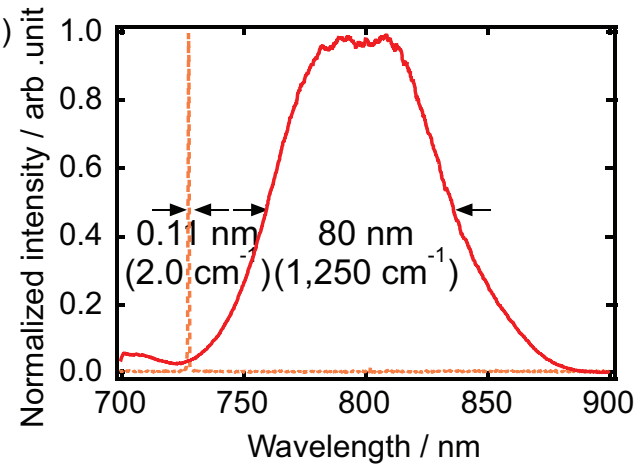

(c)

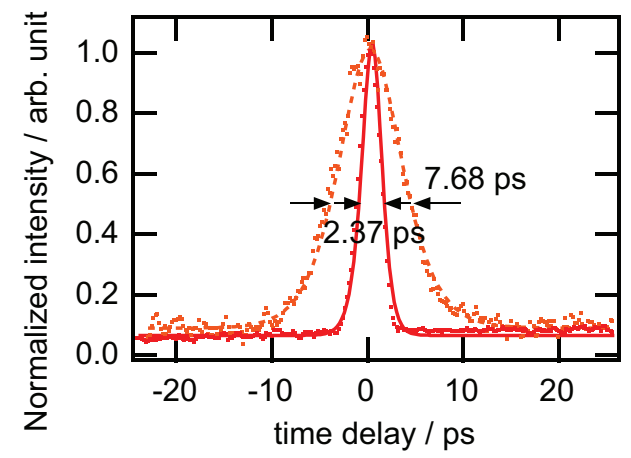

FIG. 1. Multiplex CARS microspectroscopy using scanning elliptical focal spot. (a) Schematic of the developed system. ps Ti:s $\left(\omega_{1}\right), 5$ picosecond Ti:sapphire laser; fs Ti:s $\left(\omega_{2}\right), 12$ femtosecond Ti:sapphire laser; DM, dichroic mirror; CL1 $(f=400 \mathrm{~mm})$ and CL2 $(f=50 \mathrm{~mm})$, cylindrical lenses; L1 $(f=200 \mathrm{~mm})$ and L2 $(f=200 \mathrm{~mm})$, lenses; TL $(f=200 \mathrm{~mm})$, tube lens; OL1 $(N A=0.9, \times 40)$ and OL2 $(N A=1.27, \times 60$ or $N A=1.0, \times 60$, dipping $)$, objective lenses; SP (600 grooves/mm, $500 \mathrm{~nm}$ blaze wavelength, $300 \mathrm{~mm}$ focal length), spectrometer. (b) Output spectra of laser sources. Solid orange line, $\omega_{1}$ beam. Broken red line, $\omega_{2}$ beam. FWHMs of 0.11 $\mathrm{nm}\left(\omega_{1}\right)$ and $80 \mathrm{~nm}\left(\omega_{2}\right)$ correspond to spectral resolution of $2.0 \mathrm{~cm}^{-1}$ and bandwidth of 1250 $\mathrm{cm}^{-1}$, respectively. (c) Normalized intensity auto-correlation signals of $\omega_{1}$ beam (orange dots) and $\omega_{2}$ beam (red dots). Solid $\left(\omega_{1}\right)$ and broken $\left(\omega_{2}\right)$ lines are fitting results with sech ${ }^{2}$ function. FWHMs of $7.68 \mathrm{ps}\left(\omega_{1}\right)$ and $2.37 \mathrm{ps}\left(\omega_{2}\right)$ correspond to pulse durations of 4.99 ps and $1.54 \mathrm{ps}$, respectively. (d) Detail view of elliptical focus shaping. 
(a)

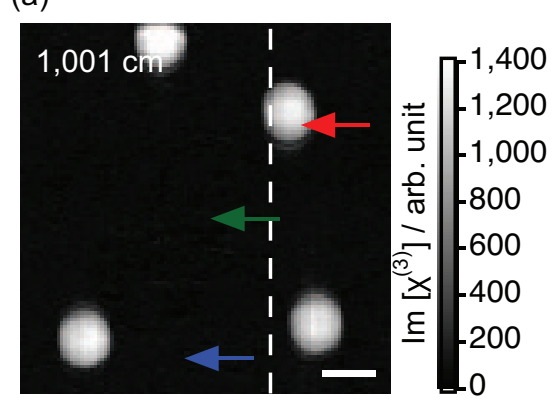

(d)

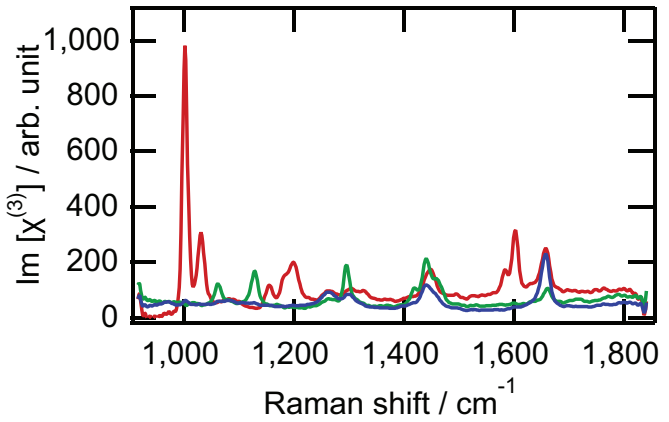

(b)

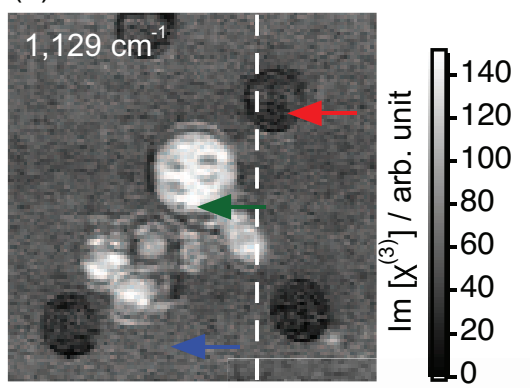

(c)

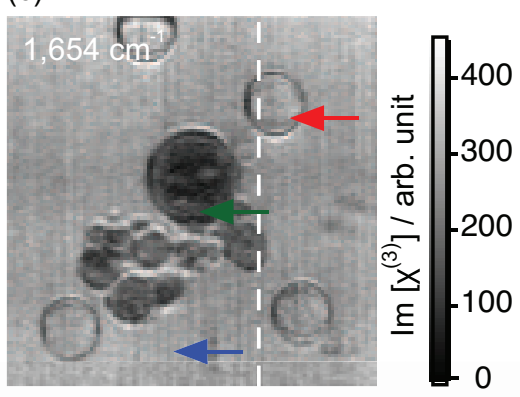

(e)

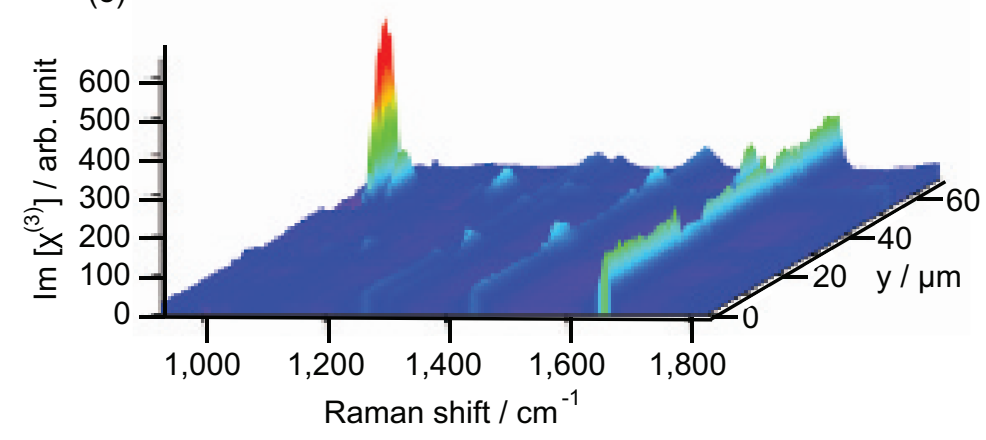

FIG. 2. Ultrahigh-speed hyperspectral CARS imaging $(34,139$ spectra/s) of a mixture of polystyrene and polyethylene beads in liquid linoleic acid. (a) $\operatorname{Im}\left[\chi^{(3)}\right]$ image at $1,001 \mathrm{~cm}^{-1}$ (phenyl ring breathing mode of the polystyrene); (b) at $1,129 \mathrm{~cm}^{-1}(\mathrm{C}-\mathrm{C}$ stretching mode of the polyethylene); (c) at $1,654 \mathrm{~cm}^{-1}(\mathrm{C}=\mathrm{C}$ stretching mode of the linoleic acid $) .(\mathrm{d}) \operatorname{Im}\left[\chi^{(3)}\right]$ spectra at arrowheads of corresponding color shown in (a-c). (e) a spectral profile on the white dashed line in (a-c) observed at $7.5 \mathrm{~ms} /$ line. The size of the hyperspectral image was $256_{(\mathrm{x})} \times 256_{(\mathrm{y})} \times 512_{(\text {spectrum })}$ pixels. Total acquisition time of hyperspectral image was $1.92 \mathrm{~s}$. The scale bar represents $10 \mu \mathrm{m}$. 

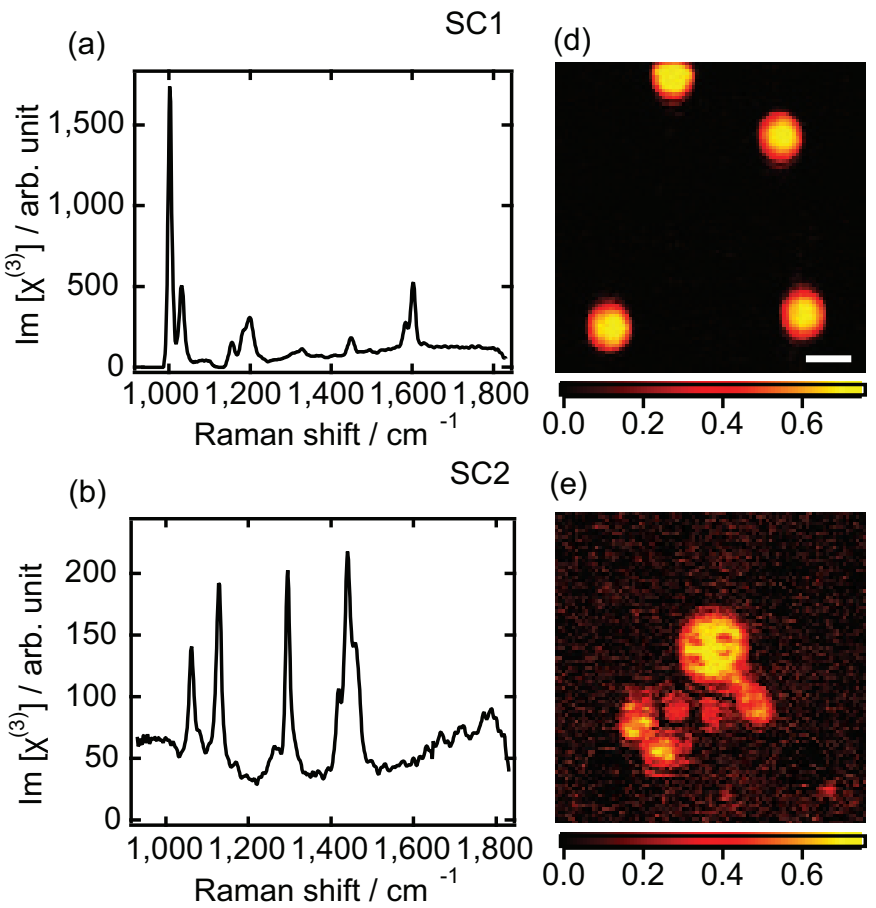

(e)

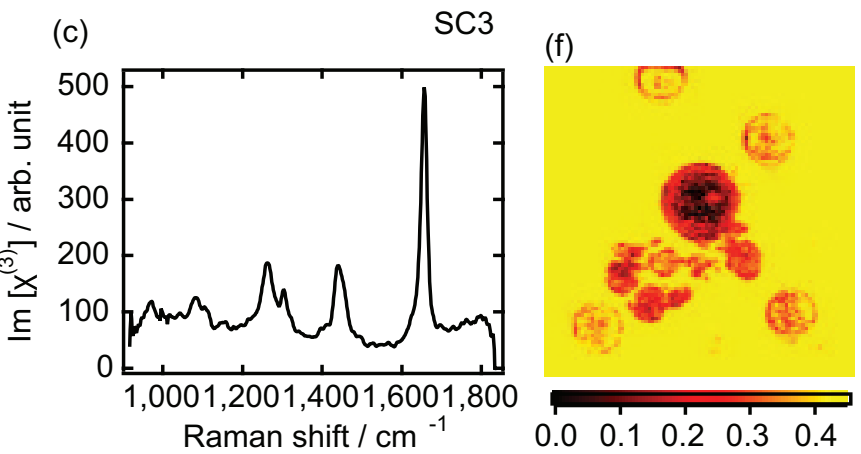

FIG. 3. MCR-ALS analysis of the hyperspectral CARS image of a mixture of polystyrene and polyethylene beads in liquid linoleic acid. (a-c) decomposed spectral components (SC1-3). (d-f) Concentration distributions of SC1-3. The scale bar represents $10 \mu \mathrm{m}$. 

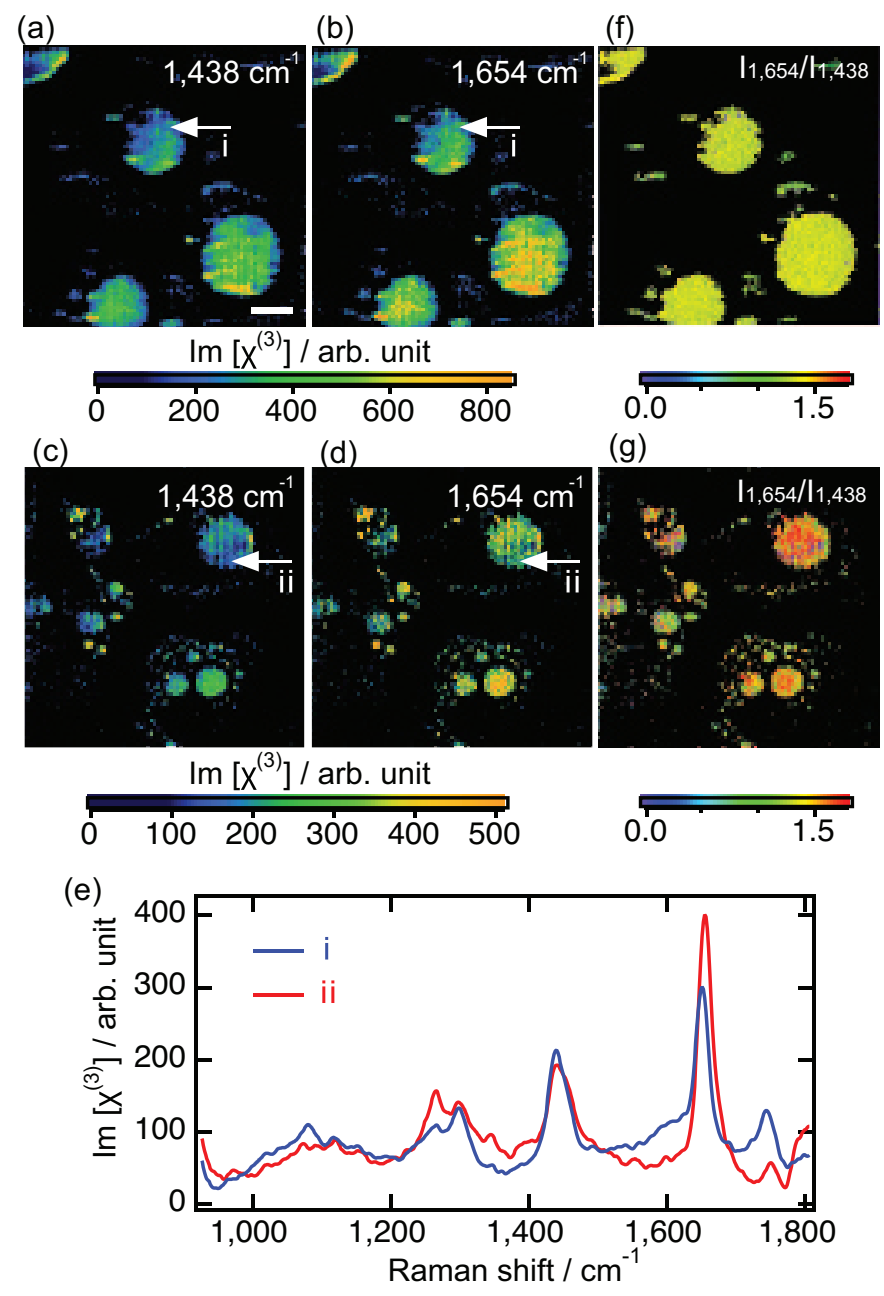

FIG. 4. Ultrahigh-speed hyperspectral CARS imaging of living 3T3-L1 adipocytes with acquisition rate of 34,139 spectra/s. The adipocytes were incubated in media to which were added (a, b, f) oleic acid or (c, d, g) linoleic acid. (a, c) $\operatorname{Im}\left[\chi^{(3)}\right]$ image at $1,438 \mathrm{~cm}^{-1}$. (b, d) $\operatorname{Im}\left[\chi^{(3)}\right]$ image at 1,654 $\mathrm{cm}^{-1}$. (e) $\operatorname{Im}\left[\chi^{(3)}\right]$ spectra at arrowheads shown in (a-d (( i ), blue; (ii), red)). (f, g) Intensity ratio of the $\operatorname{Im}\left[\chi^{(3)}\right]$ images at $1,438 \mathrm{~cm}^{-1}$ and $1,654 \mathrm{~cm}^{-1}$. The image size was $256_{(\mathrm{x})} \times 256_{(\mathrm{y})} \times 512_{(\text {spectrum })}$ pixels, and the total acquisition time was $1.92 \mathrm{~s}$. The scale bar represents $10 \mu \mathrm{m}$. 
(a)

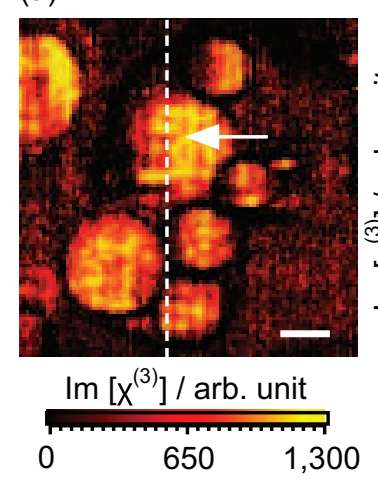

(c) (b)
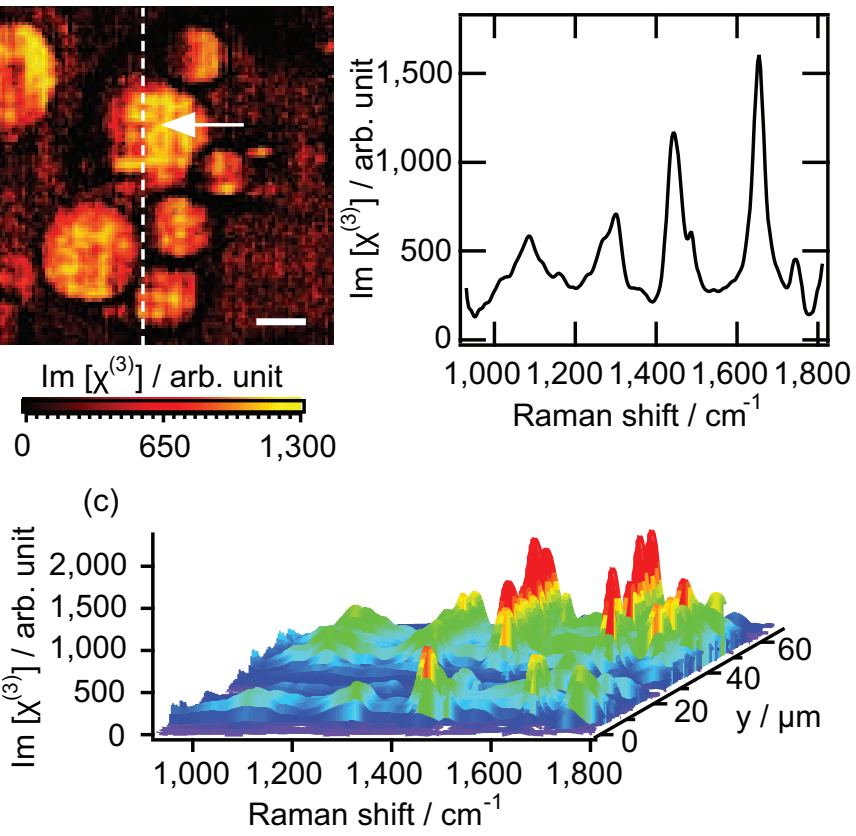

FIG. 5. Ultrahigh-speed hyperspectral CARS imaging of living 3T3-L1 adipocytes with total acquisition time of $0.54 \mathrm{~s}$. (a) $\operatorname{Im}\left[\chi^{(3)}\right]$ image at $1,438 \mathrm{~cm}^{-1}$. (b) $\operatorname{Im}\left[\chi^{(3)}\right]$ spectrum at arrowheads of corresponding color shown in (a). (c) Spectral profile on the white dashed line in (a) observed at $4.2 \mathrm{~ms} /$ line. The image size was $128_{(\mathrm{x})} \times 128_{(\mathrm{y})} \times 256_{(\text {spectrum) }}$ pixels, and the acquisition rate was $30,139 \mathrm{spectra} / \mathrm{s}$. The scale bar represents $10 \mu \mathrm{m}$. 
(a)

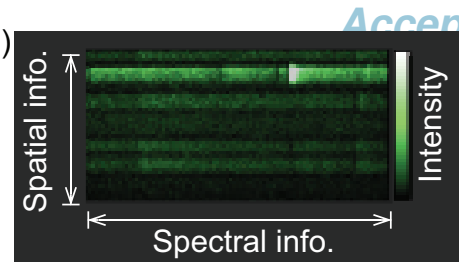

oted to I Chdi2 in

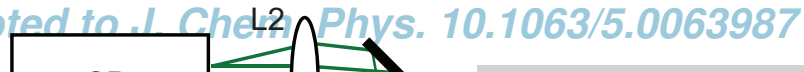

$\stackrel{\dot{c}}{\frac{5}{2}}$

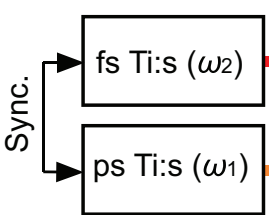

(d)

\section{EM- CCD} Ext. trig. ctrl. $\mathrm{STG}$

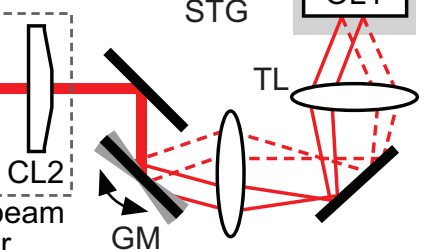
shaper $-\omega_{1}$ laser $-\omega_{2}$ laser - CARS signal

(b)

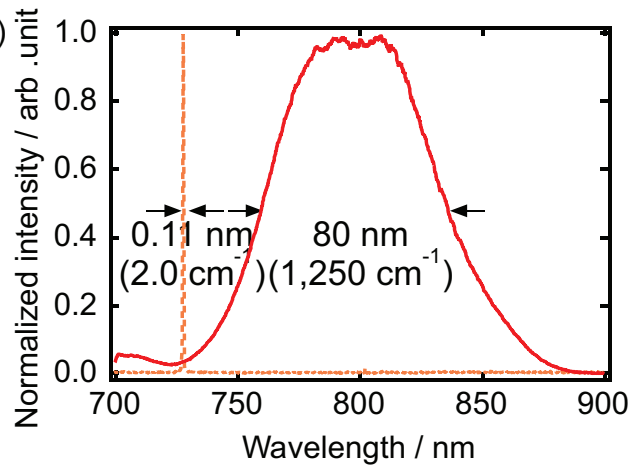

(c)

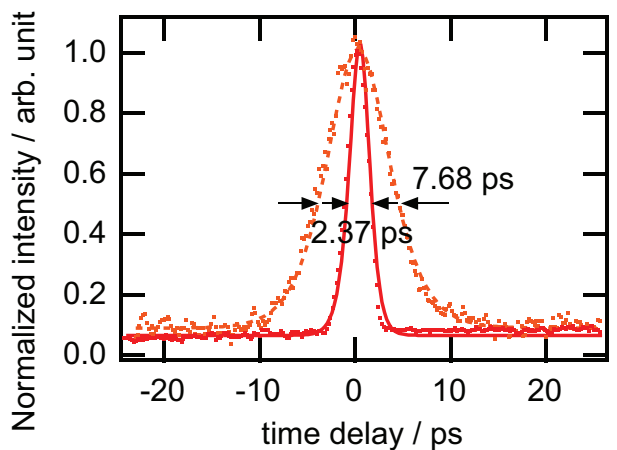


$1,438 \mathrm{~cm}^{-1}=1,654 \mathrm{~cm}^{-1}$

$|1,654 /|_{1,438}$
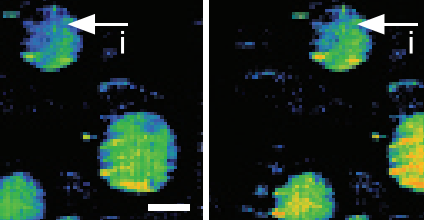

$\operatorname{Im}\left[\mathrm{x}^{(3)}\right] /$ arb. unit

\begin{tabular}{llll}
\hline 000 & 400 & 600 & 800
\end{tabular}

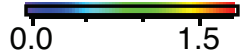

(c)

$\operatorname{Im}\left[\mathrm{x}^{(3)}\right] /$ arb. unit

(d)

*) $1,438 \mathrm{~cm}^{-1}$

$\begin{array}{llllll}0 & 100 & 200 & 300 & 400 & 500\end{array}$ (g)

$1,654 \mathrm{~cm}^{-1}$
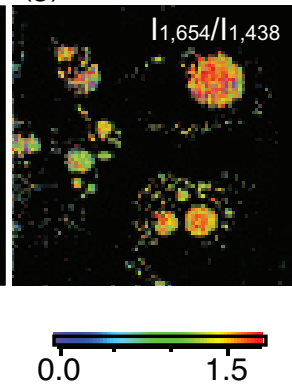

(e)

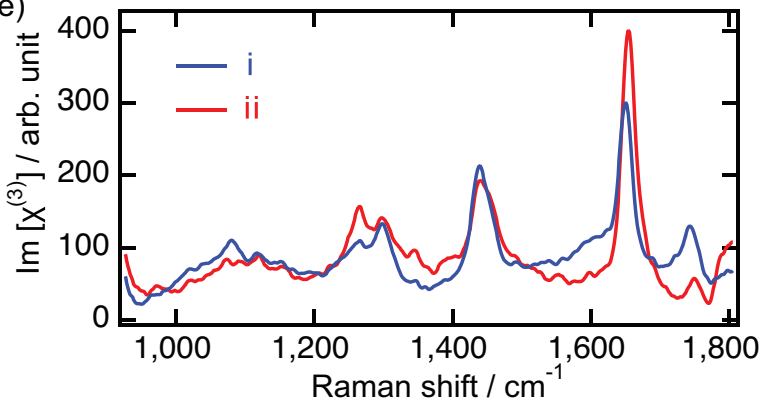


